\title{
Agitated Depression; The Challenges in Management and the Role of Maintenance Electroconvulsive Therapy
}

\author{
V.Janaki ${ }^{1}$, W.Suzaily ${ }^{2}$ \\ ${ }^{1}$ Faculty of Medicine and Health Sciences, UCSI University \\ ${ }^{2}$ Department of Psychiatry, Universiti Kebangsaan Malaysia Medical Centre
}

\section{ABSTRACT}

This case report highlights the challenges encountered and the role of maintenance electroconvulsive therapy (ECT) in the management of a depressed patient who presented with symptoms of agitation. Despite on an adequate dosage and duration of medications, the patient showed poor improvement and persistent agitation. Upon commencing maintenance ECT in addition to the pharmacotherapy, the patient's condition markedly improved. As conclusion, maintenance ECT may be another option in managing agitated depression.

KEYWORDS: agitated depression, electroconvulsive therapy, antidepressants

\section{INTRODUCTION}

Diagnosing and managing agitated depression poses a lot of challenge in clinical practice for many years. Not only the nosologic classification is debatable ${ }^{1}$, numerous issues has also been encountered in choosing the right pharmacological treatment for symptom remission. ${ }^{1}$ Even though electroconvulsive therapy (ECT) has repeatedly been demonstrated as an extremely effective acute treatment for major depressive episodes ${ }^{2}$ and also as maintenance ${ }^{2}$, its role in the management of depression with agitation has not been widely discussed. This case illustrates the problems encountered in the management and the successful use of maintenance ECT in treating a case of agitated depression.

\section{CASE REPORT}

Miss L, a 60 years old Chinese lady with underlying hypertension presented to our unit for the first time in 2008 at the age of 54 years. She had persistent low mood, anhedonia, feeling of hopelessness, poor sleep, difficulty in concentrating due to excessive

Corresponding Author:

Dr. Suzaily Wahab

Department of Psychiatry,

Universiti Kebangsaan Malaysia Medical Centre

(UKMMC), 56000 Cheras,

Kuala Lumpur, Malaysia.

Tel No: +603-91456142

Email: suzailywhb@yahoo.com worries, fear of something bad going to happen, irritability and inner agitation for duration of eight months. She was apparently well before the first presentation. There was no past psychiatric history and no family history of psychiatric illness. She was prescribed with antidepressant Mirtazapine $30 \mathrm{mg}$ but defaulted follow up.

A year later, Miss $L$ presented to our unit with similar symptoms for a month. She was treated as an inpatient and was started on Mirtazapine $30 \mathrm{mg}$. Since there was only minimal improvement in her mood and she was still irritable and anxious, a course of ECT-8 sessions was given in addition to the antidepressant. Some resolutions of symptoms were noted and she was afterwards discharged with Mirtazapine 45mg and given outpatient appointment. During her follow ups, the depressive symptoms such as low mood, anhedonia and feeling of hopelessness improved to a certain extent but her agitation and restlessness persisted.

Subsequently, she had poor compliance to medication resulting in another relapse of symptoms. She was admitted in April 2012 due to having suicidal thoughts with restlessness and agitation. Olanzapine $10 \mathrm{mg}$ was added on as an adjunct since she did not show much improvement despite on maximum dose of Mirtazapine. As the addition of antipsychotic also failed to provide further improvement, a course of ECT-12 sessions was given. The agitation resolved 
rapidly the third ECT session onwards. She became calmer and was no longer seen pacing around the ward following which she was discharged home.

Surprisingly, just two days following discharge, she attempted suicide when she tried to get herself knocked down by a moving car on the road. She described that her agitation had again worsened and became intolerable that resulted in her action. She was again readmitted and another session of ECT was commenced. The cycle of partial remission and relapse continued for over a year and ECT was continued to be given each time her condition worsened but the effect seemed to be short-lived. A trial of monthly ECT was later on started but the outcome was still unsatisfactory.

In November 2013, ECT was discontinued and she was only managed with pharmacotherapy at another hospital because family members felt that improvement with current maintenance ECT was unsatisfactory. This clearly resulted in further worsening of her agitation and restlessness. The patient was then brought back by her family to our psychiatry unit in April 2014. A course of ECT-6 sessions was given followed by maintenance ECT monthly. Post ECT, the family members were asked to closely observe the patient's mood change and any signs of increased agitation before commencement of the next ECT. The family reported a return of agitation just two weeks post ECT, therefore, since June 2014 the ECT was rescheduled to be given two weekly. With the two weekly ECT, patient seemed calmer, and no more agitation or suicidal impulse was observed. The patient admitted that she was happy and satisfied with the two weekly ECT and wished to continue with the same treatment.

\section{DISCUSSION}

There have been numerous debates on the classification of agitated depression for many years. ${ }^{1}$ Agitated depression was a specific subtype of major depressive disorder in Research Diagnostic Criteria (RDC); the precursor of the official American Psychiatric classification. In DSM-IV-TR and in ICD-10, agitated depression is discussed under both bipolar and unipolar major depressive disorders, without any subtyping or specifier. Nevertheless, in DSM-5, the agitation criteria had been totally omitted from the major depressive disorder diagnosis, posing further difficulties in classifying agitated depression. ${ }^{3}$

In line with the ongoing debate pertaining to the nosologic status of agitated depression, several challenges were identified as we managed this case. Among the challenges encountered were the issues of making a correct diagnosis and in optimizing her treatment. Several diagnoses were considered in this case which included major depressive disorder with anxious distress or major depressive disorder with mixed specifier. There was also a need to rule out the possibility of akathisia induced by her medications. The normal radiological findings indicated that organic causes were unlikely.

Severe depressive symptoms and inner unrest are the essential elements of agitated depression. This inner agitation may also appear as irritability or unprovoked rage ${ }^{4}$ and may increase the risk of suicide in depression. ${ }^{5}$ In this case, ascertaining whether the marked restlessness and impulsivity was in fact related to her depression as opposed to akathisia was challenging since the underlying causes of akathisia are multifactorial. Akathisia can be caused not only by antipsychotics, but also antidepressants, including Mirtazapine ${ }^{6}$ and the combination of both. In this case, a trial period of adding a benzodiazepine and reducing the dose of antipsychotic did not relieve her agitation. This lack of improvement suggested to us that it was less likely to be medication related akathisia.

Koukopoulos et al. ${ }^{4}$ has stated that antipsychotics, antiepileptics and lithium are the first-line pharmacological treatment for agitated depression. Mirtazapine was prescribed as a first choice antidepressant in Miss $\mathrm{L}$ due to its sedative properties. She developed inner agitation which lead to poor sleep. Furthermore, a review by Alam et $\mathrm{al}^{7}$ demonstrated that Mirtazapine can lead to rapid and sustained improvement in depressive symptoms. It is effective in subgroups of depressed patients, particularly in anxious patients and those with geriatric depression.

The recognition of depression with anxious distress or agitated depression is also important due to differing treatment strategies/ approaches compared to the standard management. Koukopoulos et $\mathrm{al}^{4}$, reported that in cases of agitated depression, antidepressants can worsen the 
agitation and the patient's suffering. Initiating antidepressants in those with agitated depression was also found to be associated with the emergence of psychotic symptoms with suicidal ideas and impulses. ${ }^{4}$ This issue was clearly highlighted by this case; the patient had multiple episodes of suicidal attempts due to inability to tolerate the agitation. The addition of antipsychotic $\overline{-}_{-}$did not achieve desired outcome, resulting in the consideration of ECT as the next option.

In general, several publications support the use of ECT under the circumstances of increased clinical urgency, intolerance to psychotropic drugs, failure of drug therapy or patient's preference. ${ }^{8,9}$ "Ms L's clinical response to ECT was remarkable in that her agitation and the suicidal impulses improved significantly even after a few sessions. We however noted that the favourable effects were short lived, necessitating Ms $\mathrm{L}$ to be offered maintenance ECT. As illustrated in our case, the of the two-weekly maintenance ECT to the pharmacotherapy had successfully controlled the agitation and distress in this patient. We observed that when the intervals between ECT sessions were longer than 2 weeks, Ms L's agitation recurred. The same issue was also reported by Zisselman et al. ${ }^{10}$ who did a case series of three patients with various mood disorders requiring weekly ECT treatments for several years. All attempts to lengthen the gaps between ECT sessions led to rapid decompensation. ECT also has a role in modulating the altered brain chemistry in patients with depression whereby it affects the expression and release of various brain neurochemicals such as major neurotransmitters, hormones, transcription factors and neurotrophic factors. $^{11,12}$

\section{CONCLUSION}

In conclusion, the presence of agitation in cases of depression needs to be fully identified, investigated and treated appropriately as it may lead to impulsive behaviour and suicidal attempts. Antidepressant alone has a limited role and further promising outcome may be seen by using maintenance ECT as an option in the treatment of major depressive disorder with mixed features especially in addressing the severe agitation.

\section{REFERENCES}

1. Akiskal HS, Benazzi F, Perugi G, et al. Agitated "unipolar" depression re-conceptualized as a depressive mixed state: implications for the antidepressant-suicide controversy.J Affect Disord 2005;85:245-58.

2. Petrides $\mathrm{G}$, Tobias KG, Kellner $\mathrm{CH}$, Rudorfer $M V$. Continuation and mainte-

nance electroconvulsive therapy for mood disorders: review of the literature. Neuropsychobiology, 2011;64:129-140.

3. Koukopoulos A, Sani G, Ghaemi SN. Mixed features of depression: why DSM-5 is wrong (and so was DSM-IV). Br J Psychiatry 2013;203:3-5.

4. Koukopoulos A, Sani G, Koukopoulos AE, et al. Melancholia agitata and mixed depression. ActaPsychiatrScand 2007; 115 (Suppl. 433): 5057.

5. Angst J, Angst F, Stassen HH. Suicide risk in patients with major depressive disorder. J Clin Psychiatry 1999; 60 (Suppl 2):57-62.

6. Markoula S, Konitsiotis S, Chatzistefanidis D, et al. Akathisia Induced by Mirtazapine After 20 Years of Continuous Treatment. Clinical Neuropharmacology 2010; 33,50-51.

7. Alam A, Voronovich Z, Carley JA. A Review of Therapeutic Uses of Mirtazapine in Psychiatric and Medical Conditions. Prim Care Companion CNS Disord. 2013; 15(5).

8. Abrams R. Electroconvulsive Therapy. Oxford University Press: New York, 2002.

9. Prudic J. Electroconvulsive therapy. In: Saddock BJ, Saddock VA, editors. Comprehensive textbook of psychiatry. Eighth. Vol. 2. Philadelphia: Lippincott Williams \& Wilkins 2005.

10. Zisselman MH, Rosenquist PB, Curlik SM. Longterm weekly continuation electroconvulsive therapy: a case series. J ECT 2007;23:274-7.

11. Njau S, Joshi SH, Espinoza R, et al. Neurochemical correlates of rapid treatment response to electroconvulsive therapy in patients with major depression. J Psychiatry Neurosci. 2017;42(1):6-16.

12. Singh A \& Kar SK. How Electroconvulsive Therapy Works?:Understanding the Neurobiological Mechanisms. Clin Psychopharmacol Neurosci. 2017; 15(3): 210 221. 